\title{
Combined regenerated fibre Bragg gratings and Fabry-Perot etalons for dual strain and temperature sensing
}

Zengling Ran, Haihong Bao, Kevin Cook, Xuezhong Wu, Yunjiang Rao, et al.

Zengling Ran, Haihong Bao, Kevin Cook, Xuezhong Wu, Yunjiang Rao, John Canning, "Combined regenerated fibre Bragg gratings and Fabry-Perot etalons for dual strain and temperature sensing," Proc. SPIE 9634, 24th International Conference on Optical Fibre Sensors, 963459 (28 September 2015); doi: 10.1117/12.2194860

EDent: International Conference on Optical Fibre Sensors (OFS24), 2015, Curitiba, Brazil 


\title{
Combined regenerated fibre Bragg gratings and Fabry-Perot etalons for dual strain and temperature sensing
}

\author{
Zengling Ran ${ }^{1 \#}$, Haihong Bao ${ }^{1 \#}$, Kevin Cook $^{2}$, Xuezhong Wu${ }^{1}$, Yunjiang Rao ${ }^{1 *}$, John Canning ${ }^{2}$ \\ ${ }^{1}$ Key Laboratory of Optical Fiber Sensing \& Communications (Ministry of Education), \\ University of Electronic Science \& Technology of China, Chengdu, Sichuan 610054, China \\ ${ }^{2}$ interdisciplinary Photonics Laboratories (iPL), School of Chemistry, The University of Sydney, NSW 2006, Australia \\ *Corresponding author: yjrao@uestc.edu.cn \\ \#Co-first authors: these two authors contributed equally to this study
}

\begin{abstract}
A highly integrated fibre-optic sensor with regenerated fibre Bragg grating (RFBG) and a micro Fabry-Pérot (MFP) is proposed and demonstrated for simultaneous measurement of temperature and strain under high temperature $\left(>600{ }^{\circ} \mathrm{C}\right)$. The MFP is fabricated by using a $157 \mathrm{~nm}$ fluorine gas $\left(\mathrm{F}_{2}\right)$ laser to micromachine the core of a standard optical fibre. The RFBG is fabricated by regenerating a seed grating written over the Fabry-Pérot. Since the MFP and RFBG have different sensitivity coefficients, their combination can be used to realise simultaneous measurement of temperature and strain. It is believed that such a high-temperature strain sensor could find important applications in many areas where simultaneous measurement of temperature and strain under high temperature is required.
\end{abstract}

Key Words: Optical fiber sensors, high temperature measurement, strain gauges, regeneration, Bragg gratings, Fabry-Pérot.

\section{INTRODUCTION}

Precise strain measurement is important for many high-temperature applications, such as health monitoring of engines, aeronautic test of aircrafts and wind tunnels, and production process monitoring of composite materials [1-4]. Generally, in order to achieve accurate measurements, simultaneous measurement of temperature and strain is essential as the mechanical strain induced by thermal expansion can seriously influence the strain measurement accuracy [5-13]. Simultaneous measurement schemes based on different combinations of in-fibre Bragg grating (FBG) sensors and/or fibre-optic extrinsic Fabry-Perot interferometric (EFPI) sensors have been the subject of intense research in recent times because the thermal strain induced by temperature change for the strain sensor can be directly compensated using the temperature sensor nearby [5,7-9]. However, many of these techniques lack the ability to endure high temperature operation.

In this paper, a highly integrated sensor head, formed by overlapping a RFBG [14] on a MFP, has been demonstrated successfully for simultaneous measurement of temperature and strain. The co-location of both sensors on the same length of fibre is an important attribute when compared to simultaneous measurement methods utilising separate sensors. The micro F-P cavity was fabricated by using a $157 \mathrm{~nm}$ laser to micro-mill the core of cleaved fibre end-face before splicing to an un-milled section of cleaved fibre. The RFBG was first written over the same position of the F-P cavity and then regenerated by a thermal processing procedure. The RFBG operation was tested up to over $600{ }^{\circ} \mathrm{C}$, exhibiting a linear response to temperate and strain. The combined RFBG-MFP is subsequently characterised.

\section{EXPERIMENT}

The configuration of the sensor is illustrated in Fig. 1(a). The sensor head consists of a MFP cavity and a RFBG, with the MFP cavity located in the middle of the RFBG. The first step involved the fabrication of the MFP cavity in hydrogen-loaded fibre. A micro-hole was fabricated in the core of a cleaved fibre end-face using $157 \mathrm{~nm}$ micro-machining. The pulse energy density and pulse repetition rate were $E=26 \mathrm{~J} / \mathrm{cm}^{2}$ and $R R=20 \mathrm{~Hz}$, respectively. The machined fibre is subsequently spliced to another cleaved hydrogen-loaded fibreto form a FP cavity.

24th International Conference on Optical Fibre Sensors, edited by Hypolito José Kalinowski, José Luís Fabris, Woitek J. Bock, Proc. of SPIE Vol. 9634, 963459 • @ 2015 SPIE CCC code: $0277-786 X / 15 / \$ 18 \cdot$ doi: $10.1117 / 12.2194860$ 

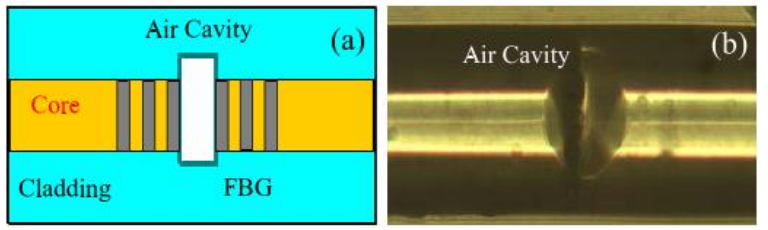

Fig. 1. (a) Structure of the MFP/RFBG; (b) photo of the MFP/SFBG sensor.

The FP cavity is shown in Fig.1 (b). Prior to grating writing, etalons are hydrogen-loaded to help the inscription of strong gratings and enable subsequent regeneration. MFP etalon samples were pressurized at $P=18 \mathrm{MPa}$ and stored at $T=80$ ${ }^{\circ}$ Cfor 48 hours before grating writing. A $193 \mathrm{~nm}$ ArF laser $\left(f=90 \mathrm{~mJ} / \mathrm{cm}^{2} ; R R=30 \mathrm{~Hz}\right)$ was used to fabricate a $1 \mathrm{~cm}$ seed grating using the direct-write method through a phase mask. Immediately after the seed grating fabrication, the grating was placed in a computer-controlled oven for regeneration. The temperature was ramped from room temperature to $950^{\circ} \mathrm{C}$ over a period of 60 mins. It was then held constant at $950{ }^{\circ} \mathrm{C}$ for 20 mins whilst the regeneration process occurred. The grating was then cooled down to room temperature over 2 hours. The final regenerated grating strength was $R \sim 30 \%$ in reflection after cooling and is shown in Fig. 2. The reflection peak sits on top of the broad F-P transmission fringes.

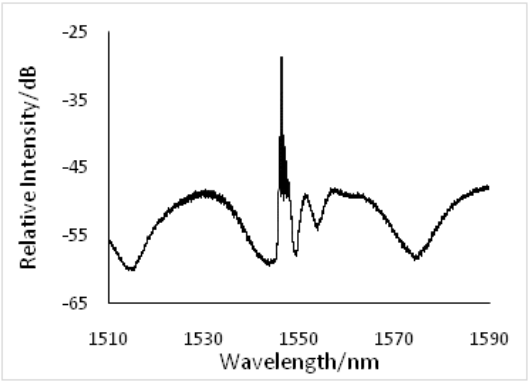

Fig. 2. Reflection spectrum of the RFBG-MFP.

The measurement principle of the RFBG-MFP sensor for simultaneous measurement of high-temperature and strain is based on the fact that the MFP and RFBG have different thermal and strain sensitivity coefficients. Both resonant wavelengths shift of the FP cavity and RFBG respond linearly to strain and temperature changes, described as:

$$
\begin{aligned}
& \Delta \lambda_{1}=\alpha_{1} \Delta T+\beta_{1} \Delta \varepsilon \\
& \Delta \lambda_{2}=\alpha_{2} \Delta T+\beta_{2} \Delta \varepsilon
\end{aligned}
$$

where $\alpha_{1}$ and $\alpha_{2}$ are the thermal coefficients of the RFBG and the FP cavity respectively.; $\beta_{1}$ and $\beta_{2}$ are strain coefficients of the RFBG and the FP cavity respectively; and $\Delta \lambda_{1}$ and $\Delta \lambda_{2}$ are the wavelength shifts of the RFBG and the FP cavity respectively. $\Delta T$ and $\Delta \varepsilon$ are temperature and strain variations, they can be obtained from equations (1) and (2):

$$
\begin{gathered}
\Delta T=\frac{\beta_{2} \Delta \lambda_{1}+\beta_{1} \Delta \lambda_{2}}{\alpha_{1} \beta_{2}-\alpha_{2} \beta_{1}} \\
\Delta \varepsilon=\frac{\alpha_{2} \Delta \lambda_{1}+\alpha_{1} \Delta \lambda_{2}}{\alpha_{2} \beta_{1}-\alpha_{1} \beta_{2}}
\end{gathered}
$$

To assess the feasibility of the FP/RFBG as a sensor, one device was placed into a high temperature furnace while each end of the fibre was fixed to two precise translation stages, with a separation of

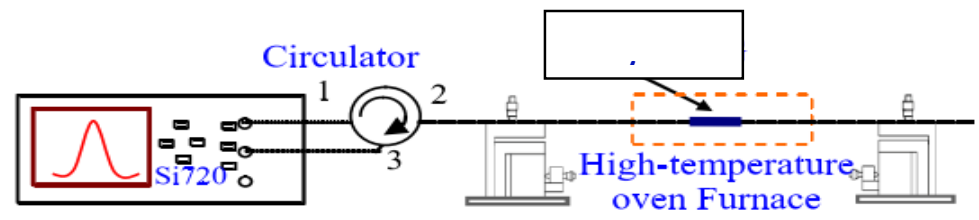

Fig. 3. The diagram of the experimental set-up. 
$72 \mathrm{~cm}$. A diagram of the experiment set up is shown in Fig. 3. The sensor head was tested from $200{ }^{\circ} \mathrm{C}$ to $620^{\circ} \mathrm{C}$. The temperature coefficients of the RFBG and the FP cavity are found to be $d \lambda d T=12.5 \mathrm{pm} /{ }^{\circ} \mathrm{C}$ and $d \lambda d T=1.2 \mathrm{pm} /{ }^{\circ} \mathrm{C}$, respectively, as shown in Fig. 4 and Fig. 5.

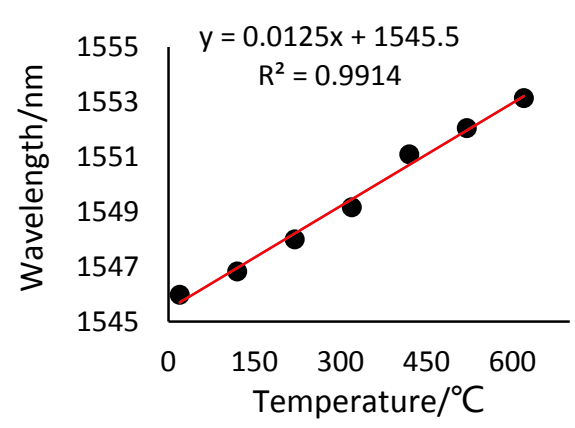

Fig.4 Thermal response of the RFBG.

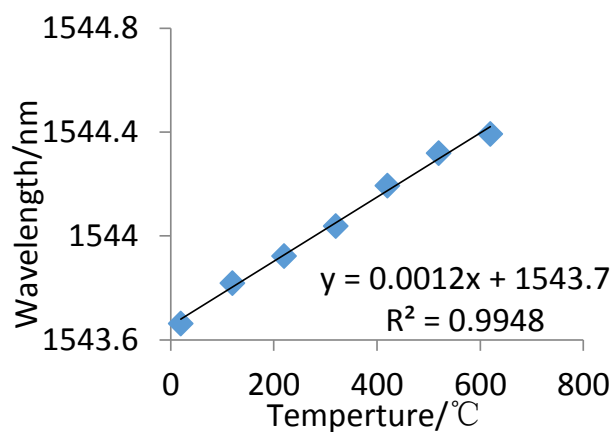

Fig.5 Thermal response of the FP cavity.

The strain responses of the RFBG and FP cavity are also tested. The strain coefficients of the MFP cavity are $d \lambda d \varepsilon=$ $3.5 \mathrm{pm} / \mu \varepsilon$ at $220{ }^{\circ} \mathrm{C}$, and $d \lambda d \varepsilon=3.6 \mathrm{pm} / \mu \varepsilon$ at $620{ }^{\circ} \mathrm{C}$ as shown in Fig. 6 . The strain coefficient of the RFBG are $d \lambda / d \varepsilon$ $=0.9 \mathrm{pm} / \mu \varepsilon$ at $220{ }^{\circ} \mathrm{C}$, and $d \lambda / d \varepsilon=1 \mathrm{pm} / \mu \varepsilon$ at $620^{\circ} \mathrm{C}$ as shown in Fig. 7.

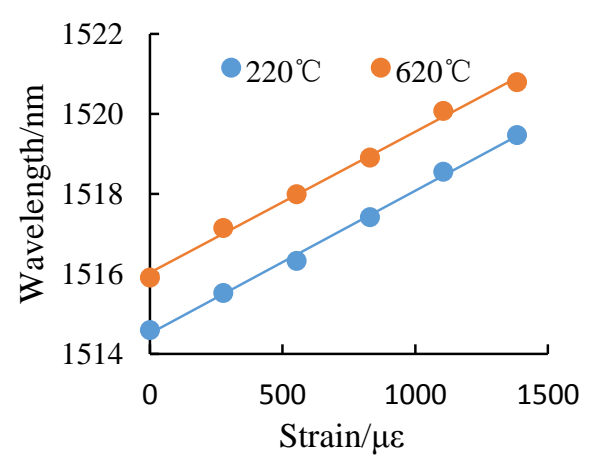

Fig. 6 The strain response of FP cavity

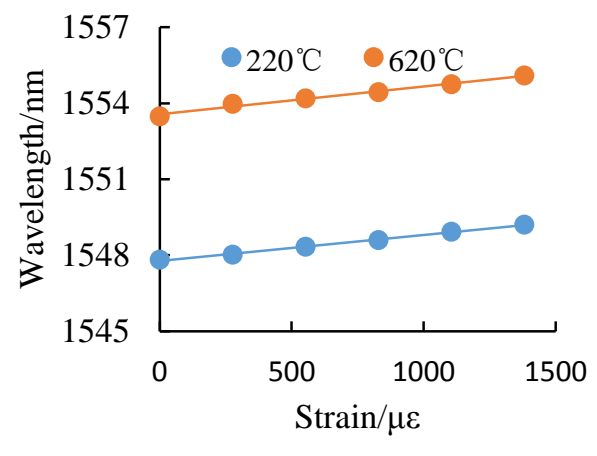

Fig. 7 The strain response of RFBG

From the results given before, simultaneous measurement of high-temperature and strain is achieved based on the complimentary properties of the RFBG and the FP sensor, where the RFBG is much more sensitive to temperature than to strain, and the FP is more sensitive to strain than to temperature.

\section{CONCLUSION}

In conclusion, a highly integrated sensor head is demonstrated by combining an in-line micro Fabry- Perot cavity fabricated by $157 \mathrm{~nm}$ laser-micromachining with a regenerated fiber Bragg grating fabricated by a $193 \mathrm{~nm}$ laser. Such a device has been used to precisely measure the temperature and strain on a local point simultaneously under high temperature. Due to its miniature size, it could be widely used in many fields such as wind tunnel tests, oil well monitoring and the monitoring of aircraft engines and other gas turbines.

\section{ACKNOWLEDGMENT}

This work was supported in part by the National Natural Science Foundation of China under Grant 51205049, Program for Changjiang Scholars and Innovative Research Team in University (PCSIRT, IRT1218), and the 111 Project (B14039), Major Equipment Projects of the Ministry of Science and Technology (M1701010112YQ2500213) and Program for New Academic Men Award of UESTC (Y03001023601017010). 


\section{REFERENCES}

[1] B. Lee, "Review of the present status of optical fiber sensors," Opt. Fiber Technol., vol. 9, no. 2, pp. 57-79, (2003).

[2] Y. J. Rao, "In-fibre Bragg grating sensors," Meas. Sci. Technol., vol. 8, no. 4, pp. 355-375, (1997).

[3] J. S. Sirkis, D. D. Brennan, M. A. Putman, T. A. Berkoff, A. D. Kersey, and E. J. Friebele, "In-line fiber etalon for strain measurement," Opt. Lett., vol. 18, no. 22, pp. 1973-1975, (1993).

[4] Z. L. Ran, Y. J. Rao, H.-Y. Deng, and X. Liao "Miniature in-line photonic-crystal-fiber etalon fabricated by $157 \mathrm{~nm}$ laser micromachining," Opt. Lett., vol. 32, no. 21, pp. 3071-3073, (2007).

[5] Y. J. Rao, P. J. Henderson, D. A. Jackson, L. Zhang, and I. Bennion, "Simultaneous strain, temperature and vibration measurement using a multiplexed in-fibre-Bragg-grating/fibre-Fabry-Perot sensor system," Electron. Lett., vol. 33, no. 24, pp. 2063-2064, (1997).

[6] H. Singh and J. S. Sirkis, "Simultaneously measuring temperature and strain using optical fiber microcavities," J. Lightw. Technol., vol. 15, no. 4, pp. 647-653, (1997).

[7] S. E. Kanellopoulos, V. A. Handerek, and A. J. Rogers, "Simultaneous strain and temperature sensing with photo generated in-fiber gratings," Opt. Lett, vol. 20, no. 3, pp. 333-335, (1995).

[8] Y. N. Tan, Y. Zhang, L. Jin, and B. O. Guan, "Simultaneous strain and temperature fiber grating laser sensor based on radiofrequency measurement,” Opt. Exp., vol. 19, no. 21, pp. 20650-20656, (2011).

[9] S. W. James, M. L. Dockney, and R. P. Tatam, "Simultaneous independent temperature and strain measurement using in-fibre Bragg grating sensors,” Electron. Lett., vol. 32, no. 12, pp. 1133-1134, (1996).

[10] Y. J. Rao, Z. L. Ran, X. Liao, and H. Y. Deng, "Hybrid LPFG/MEFPI sensor for simultaneous measurement of high-temperature and strain," Opt. Exp. vol. 15, no. 22, pp. 14936-14941, (2007).

[11] S. Triollet, L. Robert, E. Marin, and Y. Ouerdane, "Discriminated measures of strain and temperature in metallic specimen with embedded superimposed long and short fibre Bragg gratings,” Meas. Sci. Technol., vol. 22, no. 1, p. 015202, (2011).

[12] Z. Ran, C. Li, H. Zuo, and Y. Chen, "Laser-machined cascaded micro cavities for simultaneous measurement of dual parameters under high temperature,” IEEE Sensors J., vol. 13, no. 5, pp. 1988-1991, (2013).

[13] J. S. Sirkis, "A unified approach to phase-strain-temperature models for smart structure interferometric optical fiber sensors: Part 2, applications,” Appl. Opt., vol. 32, no. 4, pp. 762-773, (1993).

[14] J. Canning, S. Bandyopadhyay, P. Biswas, M. Aslund, M. Stevenson, K. Cook, "Regenerated fibre Bragg gratings", in Frontiers in Guided Wave Optics and Optoelectronics, (Ed. Bishnu Pal), IN-TECH, Vienna, Austria, European Union, (2010) and refs therein. 